J.-X. Cai

Nagoya Math. J.

Vol. 168 (2002), 93-103

\title{
NUMERICAL CRITERIA FOR CERTAIN FIBER SPACES TO BE BIRATIONALLY TRIVIAL
}

\author{
JIN-XING CAI
}

\begin{abstract}
Let $f: X \rightarrow B$ be a fiber space over a curve $B$ whose general fiber $F$ belongs to one of the following type: 1) $F$ is of general type and satisfying some mild conditions, 2) $F$ is with trivial canonical sheaf. In this note, a numerical characterization for $f: X \rightarrow B$ to be birationally trivial is given.
\end{abstract}

\section{$\S 1$. Introduction}

Let $X$ be a complex projective manifold, and $f: X \rightarrow B$ be a morphism over a smooth projective curve $B$ with connected fibers. A natural problem is to find a numerical characterization for $f: X \rightarrow B$ to be birationally trivial (see (2.1) for the definition).

When $X$ is a surface, it is well-known that, if $g(F) \geq 2, f$ is birationally trivial if and only if $q(X)-g(B)=g(F)$, where $F$ is a general fiber of $f$, $g(F)$ (resp. $g(B)$ ) is the genus of $F$ (resp. $B$ ), and $q(X)$ is the irregularity of $X$ (cf. [2]).

In this note, we consider the higher dimensional case.

For any $1 \leq i \leq \operatorname{dim} X$, let $\mathcal{H}_{X}^{i}$ be the image of the map $H^{0}\left(\Omega_{X}^{i}\right) \otimes$ $\mathcal{O}_{X} \rightarrow \Omega_{X}^{i}$, where $\Omega_{X}^{i}$ is the sheaf of holomorphic $i$-forms on $X$. Let $\operatorname{rk} \mathcal{H}_{X}^{i}$ be the rank of $\mathcal{H}_{X}^{i}$. It is easy to see that $\operatorname{rk} \mathcal{H}_{X}^{i}$ is a birational invariant. Let $h^{i, 0}(X)=\operatorname{dim} H^{0}\left(\Omega_{X}^{i}\right)$ and $p_{g}(X)$ be the geometric genus of $X$. Our main result is the following.

THEOREM 1.1. Let $X$ be a complex projective manifold of dimension $n+1(n \geq 2)$, and $f: X \rightarrow B$ be a morphism over a smooth projective curve $B$ with connected fibers. Let $F$ be a general fiber of $f$. Assume that $h^{n-1,0}(F)=0$, and that either the canonical map $\phi_{F}$ of $F$ is birational, or $\phi_{F}$ is generically finite of degree being a prime number and $p_{g}\left(\operatorname{Im} \phi_{F}\right)=0$. Then $f$ is birationally trivial if and only if $\operatorname{rk} \mathcal{H}_{X}^{n}=1$ and $h^{n, 0}(X)=p_{g}(F)$.

Received March 12, 2001.

2000 Mathematics Subject Classification: 14D05. 
Theorem 1.1 will be proved in Section 2. In Section 3 we will give some criteria for fiber spaces whose general fibers have trivial canonical sheaf to be birationally trivial.

We use standard notations as in [3] or [10].

Acknowledgements. I would like to thank Professor S.-T. Yau for his invitation to Harvard University in Spring 1998 and Morningside Center of Mathematics for providing support, where part of this work was realized. I am grateful to the referee for helpful suggestions. This work is supported by the National Natural Science Foundation of China.

\section{§2. Proof of Theorem 1.1}

2.1. A fiber space $f: X \rightarrow B$ of relative dimension $n$ is a surjective morphism between smooth projective varieties $X$ and $B$ with connected geometric fibers of dimension $n$. We say that two fiber spaces $f_{i}: X \rightarrow B_{i}$ $(i=1,2)$ are birationally equivalent if there are birational maps $\pi_{1}: X_{1} \rightarrow$ $X_{2}$ and $\pi_{2}: B_{1} \rightarrow B_{2}$ such that $f_{2} \pi_{1}=\pi_{2} f_{1}$. A fiber space $f: X \rightarrow B$ is called birationally trivial, if it is birationally equivalent to the trivial fiber space $p: F \times B \rightarrow B$, where $F$ is a general fiber of $f$ and $p$ is the projection.

2.2. Let $f: X \rightarrow B$ be a fiber space, and $F$ a general fiber of $f$. We say that $f$ has constant moduli, if any two smooth geometric fibers of $f$ are birationally equivalent.

Assume that $f$ has constant moduli and that the Kodaira dimension of $F$ is non-negative. Then $f$ admits a very concrete description, i.e., there exists a finite group $G$ acting on $F$ and on some smooth variety $\widetilde{B}$ such that $f$ is birationally equivalent to (the smooth model of) the fiber space $p:(F \times \widetilde{B}) / G \rightarrow \widetilde{B} / G$, where the action of $G$ on the production $F \times \widetilde{B}$ is compatible with the actions on each factor and $p$ is the projection to the second factor. (See [6, Theorem 2.11] or [7, Proposition 1] for a proof.)

2.3. Let $f: X \rightarrow B$ be a fiber space of relative dimension $n$, and $F$ a general fiber of $f$. In what follows we always assume that $B$ is a curve. Then $R^{n} f_{*} \mathcal{O}_{X}$ is a locally free sheaf of rank $p_{g}(F)$. By Theorem 3.1 [5], $\mathcal{O}_{B}^{\oplus} h^{0}\left(R^{n} f_{*} \mathcal{O}_{X}\right)$ is a direct factor of $R^{n} f_{*} \mathcal{O}_{X}$. By the Leray spectral sequence,

$$
h^{0}\left(R^{n} f_{*} \mathcal{O}_{X}\right)+h^{1}\left(R^{n-1} f_{*} \mathcal{O}_{X}\right)=h^{n}\left(\mathcal{O}_{X}\right) .
$$

Combining these two facts, we get $h^{n}\left(\mathcal{O}_{X}\right) \leq h^{1}\left(R^{n-1} f_{*} \mathcal{O}_{X}\right)+p_{g}(F)$. 
Notation 2.4. Let $X$ be a complex projective manifold. For any $0 \neq$ $\alpha \in H^{0}\left(\Omega_{X}^{i}\right)(1 \leq i \leq \operatorname{dim} X)$, we denote by $\mathrm{Z}(\alpha)$ the zero-locus of the holomorphic $i$-form $\alpha$.

2.5. Let $f: X \rightarrow B$ and $F$ be as in 2.3. Let $\iota$ be the embedding of $F$ in $X$. We can factor the pullback of forms under the restriction map $\iota^{*}: \Omega_{X}^{n} \rightarrow \Omega_{F}^{n}$ by

$$
\left.\Omega_{X}^{n} \stackrel{r}{\longrightarrow} \Omega_{X}^{n}\right|_{F} \longrightarrow \Omega_{F}^{n}
$$

Consider the long exact sequences associated with the exact sequences of sheaves

$$
\begin{gathered}
\left.0 \longrightarrow \Omega_{X}^{n}(-F) \longrightarrow \Omega_{X}^{n} \stackrel{r}{\longrightarrow} \Omega_{X}^{n}\right|_{F} \longrightarrow 0 \text { and } \\
\left.0 \longrightarrow \Omega_{F}^{n-1} \longrightarrow \Omega_{X}^{n}\right|_{F} \longrightarrow \Omega_{F}^{n} \longrightarrow 0
\end{gathered}
$$

Then we have that, if $h^{n-1,0}(F)=0$, then for any $0 \neq \varphi \in H^{0}\left(\Omega_{X}^{n}\right), \iota^{*} \varphi=0$ if and only if $\varphi \in \operatorname{Ker} r$, i.e., $F \subset \mathrm{Z}(\varphi)$.

2.6. Let $X$ be a complex projective manifold of dimension $n+1$ $(n \geq 2)$, with $h^{n, 0}(X) \geq 2$. Assume that there are two linearly independent $n$-forms $\varphi_{1}$ and $\varphi_{2}$ such that $\varphi_{1} \wedge \varphi_{2}=0$ in $H^{0}\left(\bigwedge^{2} \Omega_{X}^{n}\right)$. Then there exists a non-constant rational function $h$ on $X$ such that $\varphi_{2}=h \varphi_{1}$. Let $\pi: X^{\prime} \rightarrow X$ be the blowing up of the locus of indeterminacy of the rational map

$$
(1: h): X--\rightarrow \mathbb{P}^{1}
$$

and $f_{h}: X^{\prime} \rightarrow C$ the Stein factorization of $(1: h) \circ \pi$. We have that $h$ is constant along the fibers of $f_{h}$.

Lemma 2.7. Let $X$ and $f_{h}$ be as above. Then for any smooth fiber $F$ of $f_{h}$, we have

(i) $\iota_{F}^{*}\left(\pi^{*} \varphi_{i}\right)=0$ for $i=1$ and 2 , where we denote by $\iota_{F}$ the embedding of $F$ in $X^{\prime}$,

(ii) $h^{n-1,0}(F)>0$.

Proof. (i) Indeed, for any $x \in F$, let $z_{0}, z_{1}, \ldots, z_{n}$ be a set of analytic local coordinates of $X$ around $x$, such that $z_{0}$ is the pullback of a local coordinate of $C$ around the image $c$ of $F$ by $f_{h}$. Then $h$ is the pull-back of 
a non-constant holomorphic function of a neighborhood of $c$, and within an analytic neighborhood of $x$, we can write

$$
\begin{aligned}
\pi^{*} \varphi_{1} & =\sum_{i=0}^{n} A_{i} d z_{0} \wedge \cdots \wedge \widehat{d z_{i}} \wedge \cdots \wedge d z_{n} \\
\pi^{*} \varphi_{2} & =\sum_{i=0}^{n} B_{i} d z_{0} \wedge \cdots \wedge \widehat{d z_{i}} \wedge \cdots \wedge d z_{n}
\end{aligned}
$$

$\left(\widehat{d z_{i}}\right.$ indicating the omission of the $i$-th factor $d z_{i}$ ) where $A_{i}$ and $B_{i}$ are holomorphic functions of this neighborhood. Clearly we have that

$$
\iota_{F}^{*}\left(\pi^{*} \varphi_{1}\right)=\left.A_{0}\right|_{F} d z_{1} \wedge \cdots \wedge d z_{n} .
$$

Since $\varphi_{2}=h \varphi_{1}$, we have $B_{i}=h A_{i}$ for $i=0, \ldots, n$. Since $\pi^{*} \varphi_{j}$ are $d$-closed, we have

$$
\sum_{i=0}^{n}(-1)^{i} \frac{\partial A_{i}}{\partial z_{i}}=0 \text { and } \sum_{i=0}^{n}(-1)^{i} \frac{\partial B_{i}}{\partial z_{i}}=0
$$

Now

$$
\begin{aligned}
\frac{\partial h}{\partial z_{0}} A_{0} & =\frac{\partial B_{0}}{\partial z_{0}}-h \frac{\partial A_{0}}{\partial z_{0}}=\frac{\partial B_{0}}{\partial z_{0}}+h \sum_{i=1}^{n}(-1)^{i} \frac{\partial A_{i}}{\partial z_{i}} \\
& =\frac{\partial B_{0}}{\partial z_{0}}+\sum_{i=1}^{n}(-1)^{i} \frac{\partial B_{i}}{\partial z_{i}}=0 .
\end{aligned}
$$

Note that $\partial h / \partial z_{0} \neq 0$. Hence we get $A_{0} \mid F=0$.

(ii) Let $F^{\prime}$ be a general fiber of $f_{h}$ such that $F^{\prime} \not \subset \mathrm{Z}\left(\pi^{*} \varphi_{1}\right)$. Suppose that $h^{n-1,0}\left(F^{\prime}\right)=0$. Then by 2.5 we get $\iota_{F^{\prime}}^{*}\left(\pi^{*} \varphi_{1}\right) \neq 0$. On the other hand, by (i), we have $\iota_{F^{\prime}}^{*}\left(\pi^{*} \varphi_{1}\right)=0$. This is a contradiction.

The following lemma plays an important role in the proof of the Theorem 1.1.

LEMma 2.8. Let $f: X \rightarrow B$ be a fiber space of relative dimension $n \geq$ 2 , and $F$ a general fiber of $f$. Assume that rk $\mathcal{H}_{X}^{n}=1$ (where $\mathcal{H}_{X}^{n}$ is as in Section 1), and $h^{n-1,0}(F)=0$. Then $h^{0}\left(\Omega_{X}^{n}(-F)\right)=0$. 
Proof. Consider the exact sequence

$$
0 \longrightarrow H^{0}\left(\Omega_{X}^{n}(-F)\right) \longrightarrow H^{0}\left(\Omega_{X}^{n}\right) \stackrel{r}{\longrightarrow} H^{0}\left(\left.\Omega_{X}^{n}\right|_{F}\right) .
$$

Note that for any $\varphi \in H^{0}\left(\Omega_{X}^{n}\right), \varphi \in \operatorname{Ker} r$ if and only if $\mathrm{Z}(\varphi) \supset F$. Since $F$ is a general fiber of $f$, we have $\operatorname{Im} r \neq 0$ if $h^{n, 0}(X)>0$. We choose and fix a section $\varphi_{0} \in H^{0}\left(\Omega_{X}^{n}\right)$ such that $r\left(\varphi_{0}\right) \neq 0$. Now it's enough to prove that $\operatorname{Ker} r=0$. Otherwise, let $0 \neq \varphi_{1} \in \operatorname{Ker} r$. Then $\mathrm{Z}\left(\varphi_{1}\right) \supset F$. Since $\operatorname{rk} \mathcal{H}_{X}^{n}=1, \varphi_{1} \wedge \varphi_{0}=0$. So there exists a rational function $h$ on $X$ such that $\varphi_{1}=h \varphi_{0}$. Since $\mathrm{Z}\left(\varphi_{0}\right) \not \supset F$ by the choice of $\varphi_{0}, h$ vanishes on $F$.

Let $f_{h}: X \rightarrow C$ be the fiber space induced by the rational map

$$
(1: h): X--\rightarrow \mathbb{P}^{1} .
$$

By 2.7, $h^{n-1,0}\left(F_{h}\right)>0$, where $F_{h}$ is a smooth fiber of $f_{h}$. This implies $f$ and $f_{h}$ are different fibrations of $X$ since $h^{n-1,0}(F)=0$ by the assumption. So $\left.f_{h}\right|_{F}: F \rightarrow C$ is surjective. Since $h$ vanishes on $F$ and is constant on the fibers of $f_{h}$, we get that $h$ vanishes on $X$. This is a contradiction.

The following proposition is a special case of 7.2.1 in [9].

Proposition 2.9. Let $f: X \rightarrow Y$ be a morphism from a $(n+1)$-fold to a smooth projective $n$-fold. Suppose that, over a Zariski open set $U$ of $X, \varphi \in H^{0}\left(X, \Omega_{X}^{n}\right)$ can be writen locally around each point $p \in U$ as $\varphi=\alpha f^{*}(\omega)$, where $\alpha \in \mathcal{O}_{p, X}$ and $\omega \in \Omega_{f(p), Y}^{n}$. Then $\varphi=\alpha f^{*}\left(\omega^{\prime}\right)$ for some $\omega^{\prime} \in H^{0}\left(Y, \Omega_{Y}^{n}\right)$.

\subsection{Proof of Theorem 1.1}

We prove that if $\operatorname{rk} \mathcal{H}_{X}^{n}=1$ and $h^{n, 0}(X)=p_{g}(F)$, then $f$ is birationally trivial; the converse is clear since $\operatorname{rk} \mathcal{H}_{X}^{n}$ is a birational invariant of $X$ (note that $\mathrm{rk} \mathcal{H}_{X}^{n}$ equals to the greatest integer $i$ such that $\varphi_{1} \wedge \varphi_{2} \wedge \cdots \wedge \varphi_{i} \neq 0$ in $H^{0}\left(\bigwedge^{i} \Omega_{X}^{n}\right)$ for some $\left.\varphi_{1}, \ldots, \varphi_{i} \in H^{0}\left(\Omega_{X}^{n}\right)\right)$.

Let $\varphi_{0}, \varphi_{1}, \ldots, \varphi_{m}\left(m=h^{n, 0}(X)-1\right)$ be a basis of $H^{0}\left(\Omega_{X}^{n}\right)$. Since $\operatorname{rk} \mathcal{H}_{X}^{n}=1$, there are non-constant rational functions $h_{i}$ on $X$ such that $\varphi_{i}=h_{i} \varphi_{0}$ for $i=1, \ldots, m$. Consider the rational map

$$
\Phi=\left(1: h_{1}: h_{2}: \cdots: h_{m}\right): X--\rightarrow \mathbb{P}^{m} .
$$

By Bogomolov's theorem [4], $\operatorname{dim}(\operatorname{Im} \Phi) \leq n$. 
Let $F$ be a general fiber of $f$, and $\iota$ the embedding of $F$ in $X$. Since $h^{n-1,0}(F)=0$, by 2.5 ,

$$
\operatorname{Ker}\left(\iota^{*}: H^{0}\left(\Omega_{X}^{n}\right) \rightarrow H^{0}\left(\Omega_{F}^{n}\right)\right) \simeq H^{0}\left(\Omega_{X}^{n}(-F)\right) .
$$

By Lemma 2.8, $h^{0}\left(\Omega_{X}^{n}(-F)\right)=0$. So $\iota^{*}: H^{0}\left(\Omega_{X}^{n}\right) \rightarrow H^{0}\left(\Omega_{F}^{n}\right)$ is an embedding, hence an isomorphism by the assumption $h^{n, 0}(X)=p_{g}(F)$. This implies that $\left.h_{i}\right|_{F}$, the restricion of $h_{i}$ on $F$, are non-constant rational functions on $F$, and

$$
\left.\Phi\right|_{F}=\left(1:\left.h_{1}\right|_{F}:\left.h_{2}\right|_{F}: \cdots:\left.h_{m}\right|_{F}\right): X--\rightarrow \mathbb{P}^{m}
$$

is nothing but the canonical map $\phi_{F}$ of $F$. Since $\phi_{F}$ is generically finite by assumption, we get $\operatorname{dim}(\operatorname{Im} \Phi) \geq \operatorname{dim}\left(\operatorname{Im}\left(\left.\Phi\right|_{F}\right)\right)=n$. So $\operatorname{Im} \Phi=\operatorname{Im}\left(\left.\Phi\right|_{F}\right)$ is a variety of dimension $n$. This implies that $f$ has constant moduli if $\phi_{F}$ is birational. Now we show that if $\operatorname{deg} \phi_{F}$ is prime and $p_{g}\left(\operatorname{Im} \phi_{F}\right)=0, f$ also has constant moduli.

Consider the following commutative diagram

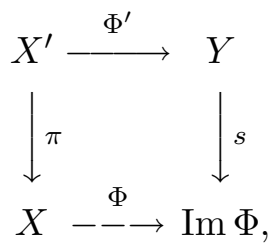

where $\pi$ is the blowing up of the locus of indeterminacy of the rational map $\Phi$ and $\Phi^{\prime}$ is the Stein factorization of $\Phi \circ \pi$. Taking the desingularisation of $Y$ instead of $Y$, we can assume that $Y$ is smooth.

Claim. $p_{g}(Y)=h^{n, 0}(X)$.

Proof of the Claim. The case when $\operatorname{dim} X=3$ is proved in [8, p. 861]; the general case can be similarly verified. Indeed, it's enough to verify that $\pi^{*} \varphi_{i}(i=0, \ldots, m)$ are pull-backs of holomorphic $n$-forms on $Y$. Since $\operatorname{Im} \Phi$ has dimension $n$ in $\mathbb{P}^{m}$, we may assume, after changing coordinates, that $z_{i}=Z_{i} / Z_{0}$ for $i=1, \ldots, n$, forms a local coordinate system at a generic point $p \in \operatorname{Im} \Phi$, where $Z_{0}, \ldots, Z_{m}$ are homogeneous coordinates of $\mathbb{P}^{m}$. Consider the compositions $g_{i}$ of $s \circ \Phi^{\prime}$ with the projection

$$
p_{i}: \operatorname{Im} \Phi \longrightarrow \mathbb{P}^{1}, \quad\left(1: h_{1}(x): h_{2}(x): \cdots: h_{m}(x)\right) \longmapsto\left(1: h_{i}(x)\right) \text {. }
$$


By blowing up if necessary, we may assume that all $g_{i}(i=1, \ldots, n)$ are morphisms. Let

$$
U=X^{\prime} \backslash \bigcup_{i=1}^{n}\left\{\text { singular fibers of } g_{i}\right\} .
$$

Let $i_{1}: F_{1} \subset X^{\prime}$ be the inclusion of a smooth fiber of $g_{1}$. Then by 2.7 , we have $\iota_{1}^{*}\left(\pi^{*} \varphi_{i}\right)=0$ for $i=0$ and 1 . Since $\varphi_{i}=h_{i} \varphi_{0}$, we get $\iota_{1}^{*}\left(\pi^{*} \varphi_{i}\right)=0$ for all $i$. It implies that around $x \in U$,

$$
\pi^{*} \varphi_{i}=\alpha_{1 i} g_{1}^{*}\left(d z_{1}\right) \wedge \tau_{1 i}
$$

where $\alpha_{1 i} \in \mathcal{O}_{x, X^{\prime}}$ and $\tau_{1 i} \in \Omega_{x, X^{\prime}}^{n-1}$. Similarly, we have

$$
\pi^{*} \varphi_{i}=\alpha_{2 i} g_{2}^{*}\left(d z_{2}\right) \wedge \tau_{2 i}=\cdots=\alpha_{n i} g_{n}^{*}\left(d z_{n}\right) \wedge \tau_{n i},
$$

where $\alpha_{2 i}, \ldots, \alpha_{n i}$ are in $\mathcal{O}_{x, X^{\prime}}$ and $\tau_{2 i}, \ldots, \tau_{n i}$ are in $\Omega_{x, X^{\prime}}^{n-1}$. This shows that around $x \in U$,

$$
\begin{aligned}
\pi^{*} \varphi_{i} & =\alpha g_{1}^{*}\left(d z_{1}\right) \wedge g_{2}^{*}\left(d z_{2}\right) \wedge \cdots \wedge g_{n}^{*}\left(d z_{n}\right) \\
& =\alpha \Phi^{\prime *}\left(\left(p_{1} \circ s\right)^{*}\left(d z_{1}\right) \wedge\left(p_{2} \circ s\right)^{*}\left(d z_{2}\right) \wedge \cdots \wedge\left(p_{n} \circ s\right)^{*}\left(d z_{n}\right)\right)
\end{aligned}
$$

for some $\alpha \in \mathcal{O}_{x, X^{\prime}}$. Now by Proposition 2.9, we have that $\pi^{*} \varphi_{i}$ are pullbacks of holomorphic $n$-forms on $Y$.

Now we continue to prove the Theorem 1.1. Let $F^{\prime}$ be the strict transform of $F$ under $\pi$. We have the following commutative diagram

$$
\begin{aligned}
& F^{\prime} \stackrel{\left.\Phi^{\prime}\right|_{F^{\prime}}}{\longrightarrow} Y \\
& \left.\downarrow \pi\right|_{F^{\prime}} \downarrow s \\
& F \stackrel{\left.\Phi\right|_{F}=\phi_{F}}{\longrightarrow} \operatorname{Im} \Phi .
\end{aligned}
$$

If $\operatorname{deg} \phi_{F}$ is prime and $p_{g}\left(\operatorname{Im} \phi_{F}\right)=0$, then $\operatorname{deg} s \neq 1$ since $p_{g}(Y) \neq$ $p_{g}\left(\operatorname{Im} \phi_{F}\right)$. So $\operatorname{deg}\left(\left.\Phi^{\prime}\right|_{F^{\prime}}\right)=1$, and we have that $f$ has constant moduli.

By $2.2, X$ is birationally equivalent to $(F \times \widetilde{B}) / G$, where $\widetilde{B}$ and $G$ are in 2.2. We claim that $|G|=1$. In fact, from

$$
h^{n, 0}(F \times \widetilde{B})=p_{g}(F)=h^{n, 0}(X)=\operatorname{dim} H^{0}\left(\Omega_{F \times B^{\prime}}^{n}\right)^{G},
$$

we get $H^{0}\left(\Omega_{F}^{n}\right)^{G}=H^{0}\left(\Omega_{F}^{n}\right)$. So $G$ induces identity on $\operatorname{Im} \phi_{F}$. This implies $\phi_{F}$ factors through $F \rightarrow F / G \rightarrow \operatorname{Im} \phi_{F}$. So we have $|G|=1$ under the condition that either $\phi_{F}$ of $F$ is birational, or $\phi_{F}$ is generically finite of degree being a prime number and $p_{g}\left(\operatorname{Im} \phi_{F}\right)=0$. 
Remark 2.11. We give some remarks about the conditions on $F$ in Theorem 1.1.

(1) If we only assume that $F$ is of general type, the question may be too general to have a positive answer. But I failed to find an example of a birationally trivial fiber space which has a birationally non-trivial smooth deformation.

(2) If $h^{n-1,0}(F) \neq 0$, the existence of non-zero global $(n-1)$-forms on $F$ makes the case more complicated (compare 2.5). Fortunately, since varieties with $h^{n-1}\left(\mathcal{O}_{F}\right)=h^{n-1,0}(F)>0$ are special in the class of $n$ dimensional varieties of general type, this is not a strong condition.

(3) Some typical examples of $n$-folds of general type with vanishing $h^{n-1,0}$ : (a) regular surfaces of general type when $n=2$, (b) smooth complete intersections in a projective space, (c) cyclic coverings of $\mathbb{C P}^{n}$ branched along a smooth divisor, and (d) products of varieties satisfying certain numerical conditions; e.g., let $F=Y \times S$, where $Y$ (resp. $S$ ) is a smooth projective $(n-2)$-fold (resp. surface) of general type satisfying one of the following conditions: (i) $p_{g}(S)=0$, (ii) $q(S)=0$ and $h^{n-3,0}(Y)=0$, or (iii) $p_{g}(Y)=0$ and $h^{n-3,0}(Y)=0$.

(4) We note that, if the canonical map $\phi_{F}$ of $F$ is generically finite, then we have either $p_{g}\left(\operatorname{Im} \phi_{F}\right)=0$ or $p_{g}\left(\operatorname{Im} \phi_{F}\right)=p_{g}(F)$ (cf. [1, Theorem 3.1]). The following example shows that the condition on $\phi_{F}$ can not be weaken.

EXAMPlE 2.12. Let $S$ be a (smooth projective) regular surface. Assume that $\phi_{S}: S \rightarrow \operatorname{Im} \phi_{S}$ is generically finite of degree 2 and $p_{g}(S)=$ $p_{g}\left(\operatorname{Im} \phi_{S}\right)$. (See [1, Proposition 3.6] for examples of such surfaces.) Let $\sigma$ be the involution of $S$ corresponding to $\phi_{S}$. Let $\widetilde{B}$ be a smooth curve with an involution $\tau$ such that $\widetilde{B} \rightarrow B:=\widetilde{B} / \tau$ is étale. Take $X=(S \times \widetilde{B}) / \mathbb{Z}_{2}$, where $\mathbb{Z}_{2}$ acts on $S \times \widetilde{B}$ by $(s, \widetilde{b}) \rightarrow(\sigma(s), \tau(\widetilde{b}))$. It's easy to check that rk $\mathcal{H}_{X}^{2}=1$ and $h^{2,0}(X)=p_{g}(S)$. But the fiber space $f: X \rightarrow B$, which is induced by the projection $S \times \widetilde{B} \rightarrow \widetilde{B}$, is not birationally trivial.

\section{§3. Miscellaneous results}

Let $F$ be a projective manifold with trivial canonical sheaf. An automorphism $\sigma$ of $F$ is said symplectic, if $\sigma$ induces trivial action on $H^{0}\left(\omega_{F}\right)$, where $\omega_{F}$ is the canonical sheaf of $F$.

THEOREM 3.1. Let $f: X \rightarrow B$ be a fiber space of relative dimension $n$ over a curve $B$, and $F$ a general fiber of $f$. Assume that $F$ is a projective manifold with trivial canonical sheaf and that $h^{n-1,0}(F)=0$ (e.g., 
an algebraic $K 3$ surface and its higher dimensional analogue, a projective Calabi-Yau manifold, etc.). Then $h^{n, 0}(X) \leq 1$, and $h^{n, 0}(X)=1$ if and only if either $f$ is birationally trivial, or $f$ is birationally isomorphic to $(F \times \widetilde{B}) / G \rightarrow \widetilde{B} / G$, where $G$ is a finite group acting on $F$ and $\widetilde{B}$ such that the action of $G$ on $F$ is symplectic and $\widetilde{B} / G \simeq B$.

Proof. $h^{n, 0}(X) \leq 1$ follows by 2.3. Now we assume that $h^{n, 0}(X)=1$. Let

$$
\Sigma=\{\text { critical points of } f\} \cup\left\{p \in B \mid f^{*} p \subset \mathrm{Z}(\varphi)\right\}
$$

where $\varphi$ be the unique holomorphic $n$-form on $X$ up to scalar multiple. Set $B^{o}=B \backslash \Sigma, X^{o}=f^{-1} B^{o}$ and $f^{o}=\left.f\right|_{X^{o}}$.

Since $p_{g}(F)=1, \mathcal{L}:=f_{*}^{o} \omega_{X^{o}}$ is an invertible sheaf. We have an exact sequence of sheaves

$$
0 \longrightarrow\left(f^{o}\right)^{*} \mathcal{L} \longrightarrow \omega_{X^{o}}
$$

So $\omega_{X^{o}}=\left(f^{o}\right)^{*} \mathcal{L} \otimes \mathcal{O}_{X^{o}}(D)$ for some non-negative divisor $D$ on $X^{o}$. From $\mathcal{O}_{F}=\left.\omega_{X^{o}}\right|_{F}=\left.\left(f^{o}\right)^{*} \mathcal{L} \otimes \mathcal{O}_{X^{o}}(D)\right|_{F}=\mathcal{O}_{F}(D)$, we have that $D$ consists of fibers of $f^{o}$. Hence $\omega_{X^{o}}=\left(f^{o}\right)^{*} \mathcal{L}^{\prime}$ for some $\mathcal{L}^{\prime} \in \operatorname{pic}\left(B^{o}\right)$.

Since $h^{n-1,0}(F)=0$ by the assumption, by 2.5 we have that for any fiber $F$ of $f^{o}, \iota^{*} \varphi \neq 0$, where $\iota$ is the embedding of $F$ in $X^{o}$. By Lemma 4.3 of [5], we get that $f^{o}$ has constant moduli.

By $2.2, X$ is birational to $(F \times \widetilde{B}) / G$, where $G$ and $\widetilde{B}$ are in 2.2. Since

$$
\operatorname{dim} H^{0}\left(\Omega_{F \times \widetilde{B}}^{n}\right)^{G}=h^{0}\left(\Omega_{X}^{n}\right)=1=h^{0}\left(\Omega_{F \times \widetilde{B}}^{n}\right),
$$

we have that either $|G|=1$ or $G$ acts trivially on $H^{0}\left(\Omega_{F}^{n}\right)$. This proves the "only if" part. The "if" part is clear.

TheOREm 3.2. Let $f: X \rightarrow B$ be a fiber space of relative dimension $n$ over a curve $B$, and $F$ a general fiber of $f$. Assume that $F$ is an Abelian variety. Then $q(X) \leq n+g(B)$, and $q(X)=n+g(B)$ if and only if either $f$ is birationally trivial, or $f$ is birationally isomorphic to $(F \times \widetilde{B}) / G \rightarrow \widetilde{B} / G$, where $G$ is a finite Abelian group acting on $F$ and $\widetilde{B}$ such that the action of $G$ on $F$ consists of translations of $F$ and $\widetilde{B} / G \simeq B$.

Proof. By the universal property of the Albanese map, we have a 
morphism $\alpha:$ Alb $X \rightarrow$ Alb $B$ such that the following diagram

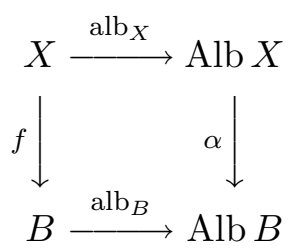

commutes. Note that $\alpha$ is a fiber bundle whose fiber $A$ is an Abelian variety of dimension $q(X)-g(B)$. Let $p$ be a general point of $B$. We have that

$$
\left.\operatorname{alb}_{X}\right|_{f^{*}(p)}: f^{*}(p) \longrightarrow A=\alpha^{*}\left(\operatorname{alb}_{B}(p)\right)
$$

is surjective since the image of $f^{*}(p)$ in $A$ generates $A$ and $f^{*}(p)$ itself is an Abelian variety. So $q(X)-g(B) \leq n=\operatorname{dim} f^{*}(p)$.

Now assume that $q(X)-g(B)=n$. Then $f$ has constant moduli since there are at most coutable Abelian varieties isogenous to a given Abelian variety. By 2.2, $X$ is birational to $(F \times \widetilde{B}) / G$, where $G$ and $\widetilde{B}$ are in 2.2 . Since

$$
\operatorname{dim} H^{0}\left(\Omega_{F \times \widetilde{B}}^{1}\right)^{G}=h^{0}\left(\Omega_{X}^{1}\right)=n+g(B)=h^{0}\left(\Omega_{F \times \widetilde{B}}^{1}\right),
$$

we have that $G$ acts trivially on $H^{0}\left(\Omega_{F}^{1}\right)$. If there is an element $\sigma \in G$ such that $\sigma$ has a fixed point, say $p \in F$, then $\sigma$ acts trivially on the tangent space $\mathrm{T}_{p} F$, since $\sigma$ acts trivially on $H^{0}\left(\Omega_{F}^{1}\right)$. This implies $\sigma=1$. So we have either $|G|=1$ or $G$ consists of translations of $F$. This proves the "only if" part. The converse is clear.

\section{REFERENCES}

[1] A. Beauville, L'application canonique pour les surfaces de type général, Invent. Math., 55 (1979), 121-140.

[2] A. Beauville, L'inegalite $p_{g} \geq 2 q-4$ pour les surfaces de type général, Appendice à O. Debarre: "Inégalités numériques pour les surfaces de type général", Bull. Soc. Math. France, 110 (1982), 343-346.

[3] W. Barth, C. Peters and A. Van de Ven, Compact complex surfaces, Ergeb. Math. Grenzgeb., 1984.

[4] F. Bogomolov, Holomorphic tensors and vector bundles on projective varieties, Math. USSR, Izv. 13 (1979), 499-555.

[5] T. Fujita, On Kaehler fibre spaces over curves, J. Math. Soc. Japan, 30 (1978), 779-794. 
[6] J. Kollár, Subadditivity of the Kodaira dimension: fibers of general type, Alg. Geom. Sendai, 1985, Adv. Studies Pure Math., 10 (1987), 361-398.

[7] M. Levine, Deformation of irregular threefolds, Lect. Notes in Math. 947, Springer (1982), pp. 269-286.

[8] T. Luo, Global 2-forms on regular 3-folds of general type, Duke Math. Jour., 71 (1993), 859-869.

[9] T. Mabuchi, Invariant $\beta$ and uniruled threefolds, J. Math. Kyoto Univ., 22 (1982), 503-554.

[10] S. Mori, Classification of higher-dimensional varieties, Proceedings of Symposia in Pure Math., 46 (1987), 269-331.

School of Mathematical Sciences

Peking University

Beijing, 100871

P. R. China

cai@math.pku.edu.cn 